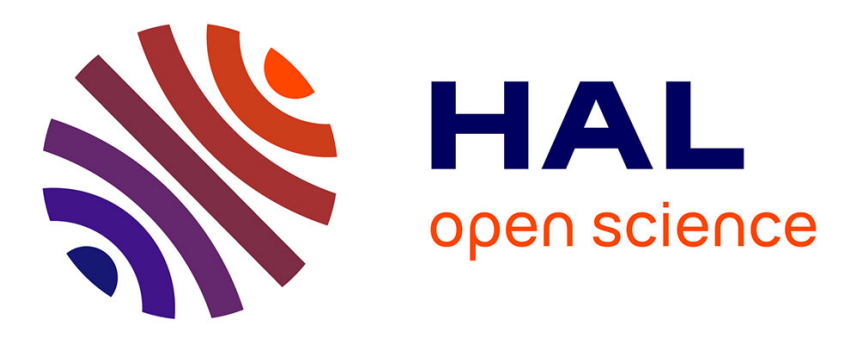

\title{
UTILISATION D'UN TUBE À ONDES PROGRESSIVES POUR LA DÉTERMINATION DU DIAGRAMME DE DIRECTIVITÉ D'HYDROPHONES DE PETITE TAILLE
}

\author{
G. Haw, P. Bigotte, J. Decarpigny, Murielle Richard
}

\section{To cite this version:}

G. Haw, P. Bigotte, J. Decarpigny, Murielle Richard. UTILISATION D’UN TUBE À ONDES PROGRESSIVES POUR LA DÉTERMINATION DU DIAGRAMME DE DIRECTIVITÉ D'HYDROPHONES DE PETITE TAILLE. Journal de Physique Colloques, 1990, 51 (C2), pp.C21299-C2-1302. 10.1051/jphyscol:19902305 . jpa-00230648

\section{HAL Id: jpa-00230648 https://hal.science/jpa-00230648}

Submitted on 1 Jan 1990

HAL is a multi-disciplinary open access archive for the deposit and dissemination of scientific research documents, whether they are published or not. The documents may come from teaching and research institutions in France or abroad, or from public or private research centers.
L'archive ouverte pluridisciplinaire HAL, est destinée au dépôt et à la diffusion de documents scientifiques de niveau recherche, publiés ou non, émanant des établissements d'enseignement et de recherche français ou étrangers, des laboratoires publics ou privés. 
UTILISATION D'UN TUBE A ONDES PROGRESSIVES POUR LA DETERMINATION DU DIAGRAMME DE DIRECTIVITE D'HYDROPHONES DE PETITE TAILLE

\author{
G. HAW, P. BIGOTTE, J.N. DECARPIGNY et M. RICHARD* \\ Laboratoire d'Acoustique, URA 253 CNRS, Institut Superieur \\ d'Electronique du Nord, 41 Boulevard Vauban, F-59046 LIIIe Cedex, \\ France \\ * Groupe d'Etude et de Recherche de Detection Sous-Marine, D.C.A.N. \\ Toulon, Le Brusc, F-83140 six Fours les plages. France
}

Résumé: Cet article décrit un tube à ondes progressives permettant de déterminer les diagrammes de directivité d'hydrophones autodirectifs en basse fréquence (300 $\mathrm{Hz}-4$ $\mathrm{kHz}$ ). Après une validation simple du dispositif, les premiers résultats sont présentés.

Abstract: This paper describes an acoustic waveguide in which the directivity patterns of directive hydrophones can be determined. The frequency range is $300 \mathrm{~Hz}-4 \mathrm{kHz}$. Test cases are first analysed and then obtained results are presented and discussed.

I N T R O D U C T I O N

La conception d'hydrophones de petite taille présentant une directivité intrinsèque en basse fréquence est intéressante pour diverses applications : réalisation d'antennes non bafflées, levée d'ambiguitté d'antennes linéaires, transmissions sous-marines, mesures en milieux confinés... Avec ces perspectives, un hydrophone à directivité cardioìde intrinsèque, dont les caractéristiques de directivité sont exploitables dans une large bande - de $100 \mathrm{~Hz}$ à $20 \mathrm{kHz}$ - a été réalisé [1]. Toutefois, si la calibration de cet hydrophone peut être conduite en bassin d'essais, de façon classique, pour des fréquences supérieures à quelques kilohertz, elle n'est possible qu'en lac pour des fréquences inférieures au kilohertz, ce qui nécessite la mise en oeuvre de moyens lourds et exclut, en outre, la possibilité de test sous pression hydrostatique élevée. Pour résoudre ce problème, un tube à ondes progressives a été conçu $[2,3]$ permettant de relever le diagramme de directivité entre $300 \mathrm{~Hz}$ et $4 \mathrm{kHz}$.

$$
\text { I- E T UDE DU T U B E A ONDES PROGR ES S I VES }
$$

Le système (fig. 1) est formé de trois parties : (i) un tube cylindrique de longueur $3 \mathrm{~m}$, de diamètre interne $192 \mathrm{~mm}$, de diamètre externe $224 \mathrm{~mm}$, comportant en son milieu une zone de mesure munie d'une ouverture circulaire, de diamètre $70 \mathrm{~mm}$, découpée dans la paroi et permettant 1'introduction des objets à caractériser, (ii) aux deux extrémités, deux transducteurs piézoélectriques de type Tonpilz qui ont fait 1'objet d'un dimerisionnement adapté [4].

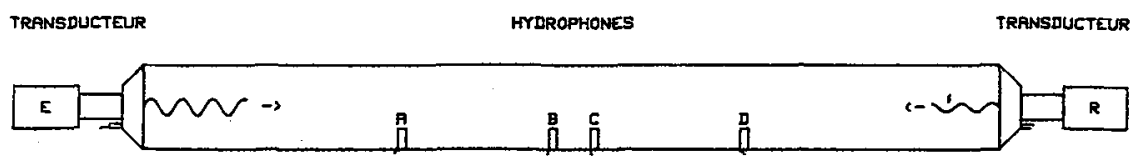

Figure 1

Les pavilions de ces transducteurs ont, en particulier, un diamètre égal à celui du tube. L'épaisseur importante de la paroi n'est toutefois pas suffisante pour considérer le tube comme infiniment rigide à toute fréquence. Aussi, un modèle a été développé prenant en compte 
la compliance de la paroi et reposant sur une approche classique due à P.M. Morse et $H$. Feshbach [5]. La compliance de la paroi entraine, de fait, une diminution de la vitesse du son dans le fluide remplissant le tube, celle-ci pouvant se calculer à partir de l'équation :

$$
\frac{\omega \cdot P_{t} \cdot h}{\rho \cdot c}-\frac{h \cdot E_{t}}{\rho \cdot c \cdot \omega \cdot a^{2}}=-\frac{1}{\tau} \cdot \frac{I_{0}(k \cdot T \cdot a)}{I_{1}(k \cdot T \cdot a)}
$$

où $\omega$ est la pulsation, $\rho$ la masse volumique du fluide, $\rho_{t}$ la masse volumique du matériau constituant la paroi, a le rayon interne du tube, h l'épaisseur de la paroi, c la célérité du son dans le fluide en champ libre, $k$ le vecteur d'onde, $E_{t}$ le module d'Young de la paroi,

$I_{i}$ la fonction hyperbolique de Bessel d'ordre $i$. La racine $T(\omega)$ étant extraite, la vitesse du son dans le fluide contenu dans le tube se déduit par la relation :

$$
c_{t}(\omega)=\frac{c}{\sqrt{1+\tau^{2}(\omega)}}
$$

Dans notre cas la vitesse évolue de $1393 \mathrm{~m} / \mathrm{s}$ à $1365 \mathrm{~m} / \mathrm{s}$ entre $200 \mathrm{~Hz}$ et $4000 \mathrm{~Hz}$, soit un écart de $8 \%$ au plus avec la vitesse en champ 1 ibre $\left(1482 \mathrm{~m} / \mathrm{s}\right.$ à $20^{\circ} \mathrm{C} \mathrm{[6]).} \mathrm{Bien} \mathrm{que} \mathrm{cet} \mathrm{écart}$ semble très faible, son influence est notable en hautes fréquences.

Pour valider le dispositif et le modèle de la relation (1), le système d'ondes stationnaires établi dans le tube par l'excitation d'un seul transducteur, $1^{\prime}$ autre se comportant comme réflecteur élastique, a été caractérisé et comparé à un modèle théorique simple.

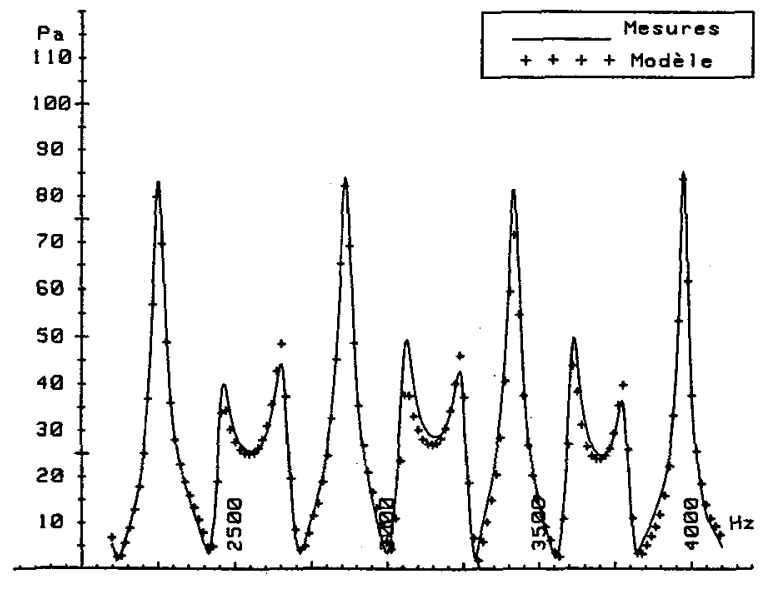

Ersquence

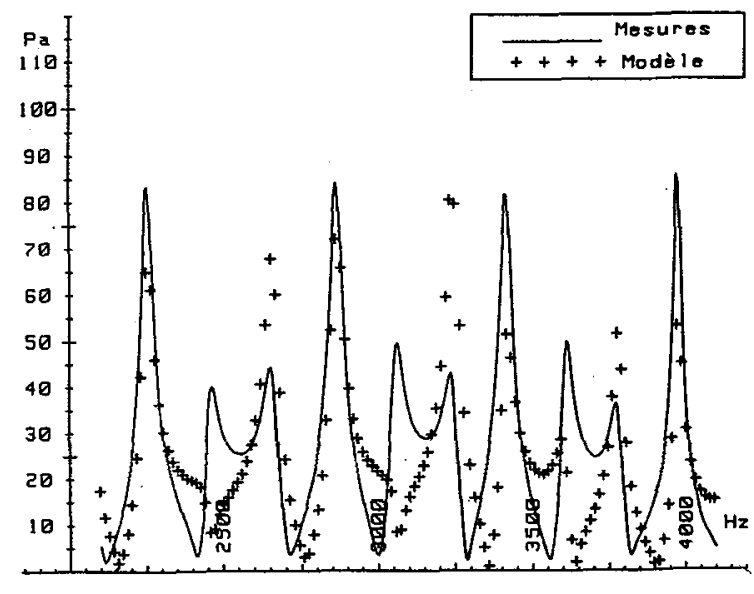

Eréquence

Pression relevée au point A du tube.

Figure 2

Figure 2bis

Ainsi, connaissant les pressions $P_{B}$ et $P_{C}$ relevées sur ces hydrophones, on peut montrer que le module de la pression en un point quelconque du tube est donné par :

$$
P(x)=P_{B} \cdot \frac{N_{1}}{D}+P_{C} \cdot \frac{N_{2}}{D}
$$


avec $N_{1}=\sin k\left(x_{C}-x\right) \cos k\left(x_{B}-x_{C}\right) \quad N_{2}=\sin k\left(x_{C}-x_{B}\right) \cos k\left(x_{B}-x\right)-\sin k\left(x_{C}-x\right)$ et $D=\sin k\left(x_{C}-x_{B}\right) \cdot \cos k\left(x_{B}-x_{C}\right)$

soit finalement :

$$
|P(x)|^{2}-\left(\frac{N_{1}}{D}\left|P_{B}\right|\right)^{2}+\left(\frac{N_{2}}{D}\left|P_{C}\right|\right)^{2}+\frac{2 N_{1} N_{2}}{D^{2}}\left|P_{B}\right|\left|P_{C}\right| \cos \left(\varphi_{C / B}\right)
$$

Les figures 2 et 2 bis comparent les résultats expérimentaux aux résultats théoriques obtenus. tenant compte ou pas de la compliance du tube. Une très bonne concordance est observée sur la figure 2, la figure 2 bis montrant bien le désaccord lorsqu'on néglige l'élasticité de la paroi.

\section{II- M E S U R E DE D I R E C T I V I T E}

Le fonctionnement du tube étant validé, la procédure de mesure de directivité est la suivante (fig. 3):

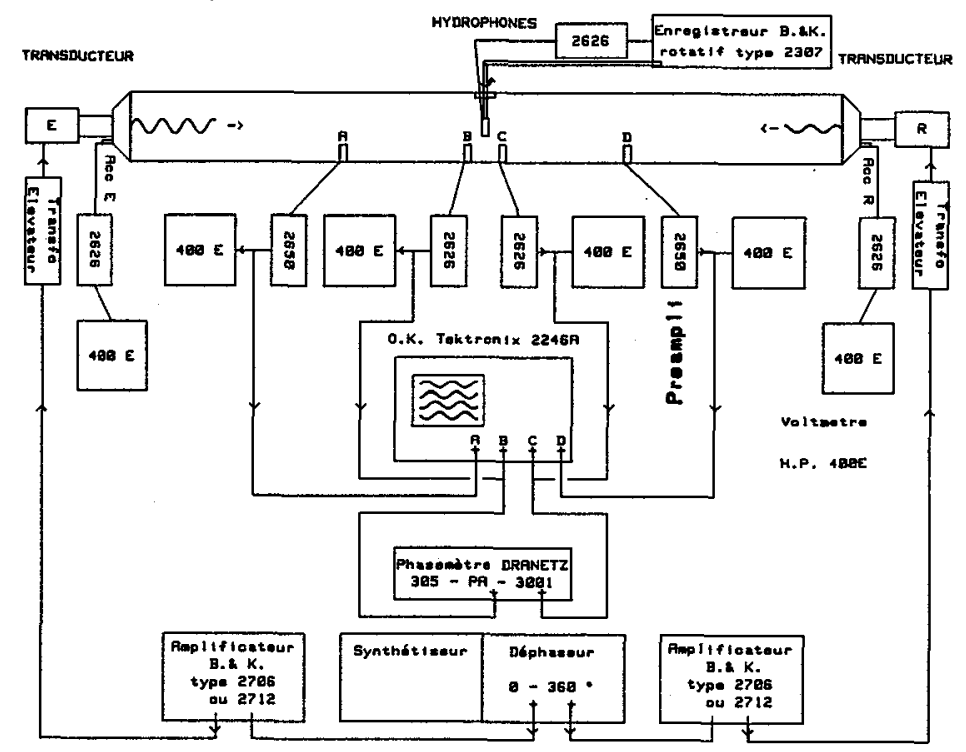

Synoptique du banc de mesure.

Figure 3

1 - on introduit l'hydrophone à caractériser, fixé sur son dispositif de rotation, par l'ouverture centrale du tube,

2 - on fixe la fréquence de travail du synthétiseur,

3 - on règle le gain des deux amplificateurs B\&K 2706 et le déphasage de leurs signaux d'attaque de telle sorte que les amplitudes sur les sondes $A, B$, C et D soient les mêmes et que le déphasage entre $B$ et $C$ corresponde à celui d'une onde progressive se propageant dan's le tube à la vitesse calculée à l'aide du modèle développé antérieurement,

4 - on relève le diagramme de directivité, en continu, à l'aide de l'enregistreur B\&K2307. 
Les résultats expérimentaux, dont les figures 4 et 5 sont un exemple, concordent bien avec des mesures faites précédemment en champ libre.
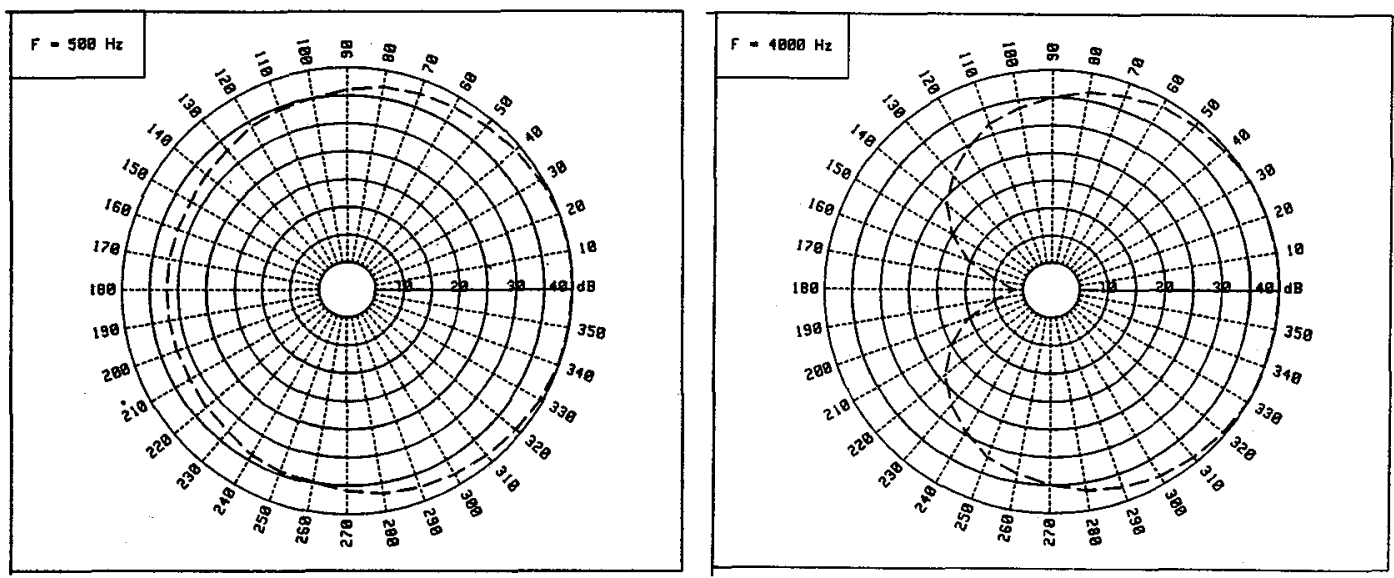

Diagrammes de directivité

Figure 4

Figure 5

Remarque: l'étape 3 est la plus délicate et un travail important reste à faire pour optimiser la procédure menant au bon réglage en un minimum de temps; toutefois, lorsque l'équilibre est atteint, il demeure parfaitement stable.

\section{O N C L U I O N}

Les résultats obtenus sont très satisfaisants et permettent d'envisager l'automatisation des réglages. Par ailleurs, il est possible de prévoir des mesures sous pression hydrostatique élevée (100 bars), pourvu que les transducteurs aient été adaptés. La transposition du dispositif à des caractérisations de matériaux absorbeurs est en cours.

\section{RE E E R N G E S}

[1] C. GRANGER, "Etude et réalisation d'hydrophones miniatures directifs ", Diplôme d'ingénieur CNAM, 1983

[2] I.G. BEATTY, J. Acoust. Soc. Am. , 39, 40, (1966)

[3] G. HAW, C. GRANGER, J. POULIQUEN, A. DEFEBVRE, "Banc d'étalonnage d'hydrophones à gradient de pression en basse fréquence", Convention C.83.48.826.033 avec la DCAN Toulon pour le GERDSM, rapport final, (1985)

[4] G. HAW, K. ANIFRANI, P. TIERCE, J.N. DECARPIGNY, " Conception d'un transducteur piézoélectrique pour tube à ondes progressives", Convention C.83.48.826.163 DCAN - Toulon (1987)

[5] P.M. MORSE, H. FESHBACH, "Methods of theoretical Physics", Ed. Mc Graw Hill, Vo1.2, 1459 , 1462, (1953)

[6] V.A. DEL GROSSO, G.W. MADER, J.Acoust.Soc.Am., 52, 1442,(1972). 